\title{
Combined analysis of EPHX1, GSTP1, GSTM1 and GSTT1 gene polymorphisms in relation to chronic obstructive pulmonary disease risk and lung function impairment
}

\author{
Ramzi Lakhdar ${ }^{\mathrm{a}, *}$, Sabri Denden ${ }^{\mathrm{a}}$, Jalel Knani ${ }^{\mathrm{b}}$, Nadia Leban ${ }^{\mathrm{a}}$, Houria Daimi ${ }^{\mathrm{a}}$, Mohsen Hassine ${ }^{\mathrm{c}}$, \\ Gérard Lefranc $^{\mathrm{d}}$, Jemni Ben Chibani ${ }^{\mathrm{a}}$ and Amel Haj Khelil ${ }^{\mathrm{a}}$ \\ ${ }^{a}$ Biochemistry and Molecular Biology Laboratory, Faculty of Pharmacy, Monastir, Tunisia \\ ${ }^{\mathrm{b}}$ Pulmonology Department, CHU Tahar Sfar, Mahdia, Tunisia \\ ${ }^{\mathrm{c}}$ Haematology Laboratory, Faculty of Pharmacy and CHU Fattouma Bourguiba, Monastir, Tunisia \\ ${ }^{\mathrm{d}}$ Institute of Human Genetics and Montpellier 2 University, Montpellier, France
}

\begin{abstract}
Smoking is considered as the major causal factor of chronic obstructive pulmonary disease (COPD). Nevertheless, a minority of chronic heavy cigarette smokers develops COPD. This suggests important contribution of other factors such as genetic predisposing. Our objective was to investigate combined role of EPHX1, GSTP1, M1 and T1 gene polymorphisms in COPD risk, its phenotypes and lung function impairment. Prevalence of EPHX1, GSTP1, M1 and T1 gene polymorphisms were assessed in 234 COPD patients and 182 healthy controls from Tunisia. Genotypes of EPHX1 (Tyr113His; His139Arg) and GSTP1 (Ile105Val; Ala114Val) polymorphisms were performed by PCR-RFLP, while the deletion in GSTM1 and GSTT1 genes was determined using multiplex PCR. Analysis of combinations showed a significant association of 113His/His EPHX1/nullGSTM1 $(\mathrm{OR}=4.07)$ and null-GSTM1/105Val/Val GSTP1 $(\mathrm{OR}=3.56)$ genotypes with increased risk of COPD (respectively P $=0.0094$ and $\mathrm{P}=0.0153$ ). The null-GSTM1/ null-GSTT1, 105Val/Val GSTP1/null GSTT1, 113His/His EPHX1/null-GSTM1 and null-GSTM1/105Val/Val GSTP1 genotypes were related to emphysema (respectively $\mathrm{P}=0.01 ; \mathrm{P}=0.009 ; \mathrm{P}=0.008$ and $\mathrm{P}=0.001$ ). Combination of 113His/His EPHX1/null-GSTM1 genotypes showed a significant association with the decrease of $\triangle \mathrm{FEV} 1$ in patients $(\mathrm{P}=0.028)$.

In conclusion, our results suggest combined EPHX1, GSTP1, GSTM1 and GSTT1 genetic polymorphisms may play a significant role in the development of COPD, emphysema and decline of the lung function.
\end{abstract}

Keywords: Chronic obstructive pulmonary disease, microsomal epoxide hydrolase, glutathione S-transferase, emphysema, genetic polymorphism

\section{Introduction}

Chronic obstructive pulmonary disease (COPD) is a devastating clinical problem and is a major cause of ill

\footnotetext{
* Corresponding author: Ramzi Lakhdar, Biochemistry and Molecular Biology Laboratory, Faculty of Pharmacy, 1, Av. Avicenne, 5019 Monastir, Tunisia. Tel.: +216 73461000; Fax: +216 73461830; E-mail: lakhdarramzi@yahoo.fr.
}

health increasing in many parts of the world. It is characterized by airflow obstruction due to chronic bronchitis, emphysema and/or small airways disease [1].

Despite that the most important risk factor for the development of COPD is cigarette smoking, it has been estimated that only 10-20\% of chronic heavy smokers will ever develop symptomatic COPD, which indicates an additional genetic or environmental contribution $[2,3]$. Factors, such as childhood viral respiratory 
infections, environmental and occupational pollution, certainly play a role in determining this susceptibility. Moreover, there is evidence that genetic susceptibility is of major importance. Several candidate genes that have been found to be involved in antiproteolysis, metabolism of toxic substances in cigarette smoke, the inflammatory response to cigarette smoke, and the efficiency of mucociliary clearance in the lung are suspected to impart in this pathogenesis [4,5]. GlutathioneS-transferases (GSTM1, T1 and P1) and microsomal epoxide hydrolase (EPHX1) were previously studied in relationship with COPD [6-14].

GST is a complex supergene family of soluble isozymes which catalyse the nucleophilic attack of glutathione on a wide range of hydrophobic electrophiles [15]. EPHX1 enzyme is an important phase II biotransformation enzyme, catalyzing the hydrolysis of various epoxides and reactive epoxide intermediates into less reactive and more water-soluble dihydrodiols [16]. Genetic polymorphisms in these two detoxifying and antioxidant enzymes can be important markers as they might affect the function of proteins and significantly influence the detoxification and oxidative stress, thereby playing a crucial role in the disease.

The two polymorphic EPHX1 (1q42.1) alleles in exon 3 (Tyr113His) and exon 4 (His139Arg) are associated with altered EPHX1 activity [17]. Four groups of putative EPHX1 phenotypes having different activities (fast, normal, slow and very slow) were reported [6] and the enzyme has been postulated as a possible determinant of COPD susceptibility, but with controversial results [6-14].

The GSTM1 (1p13) and the GSTT1 (22q 11.2) genes, encoding for respectively a mu- GST isoenzyme and a theta-GST isoenzyme, can be deleted causing a lack of the respective enzyme function. GSTP1 (11q3) encodes for a pi-GST isoenzyme heavily expressed in the digestive and respiratory epithelium, is found in humans in two allelic forms with an A to G substitution at exon 5 causing an 105Ile to105 Val replacement within the active site of the protein and in exon 6 changing alanine (Ala) to valine at the amino acid position 114 by a $\mathrm{C}$ to $\mathrm{T}$ substitution [18]. The GSTP1 105Ile/Val substitution is located near the substrate-binding site, leading to a different enzyme activity depending on the type of chemical reaction [18]. These GST polymorphisms have been investigated in relation to COPD, but without definitive conclusions [19-26].

As a complex polygenic disease, in COPD, it is likely that the operation of multiple genes is necessary and that susceptibility to disease depends on the coincident actions of several genetic events due to polymorphisms. Moreover, expression of different gene combinations probably affects the heterogeneous histopathological and clinical profile of COPD seen among individuals.

One of the possible approaches to understanding the influence of genetic factors on COPD, as well as their mutual interactions, is to assess the combined genotypes effect. GSTM1, GSTT1, GSTP1 [27,28] and EPHX1 [29] genotypes were individually studied in relationship with COPD in our sample. We aimed, in this present investigation, to analyse the EPHX1 and GST M1, T1 and P1 genotypes in combination in order to elucidate the association between the various genotype combinations and development of COPD in our population. We furthermore attempted by cross-sectional and longitudinal studies to examine the differences in COPD clinical characteristics associated to these polymorphisms.

\section{Material and methods}

\subsection{Study populations and sample processing}

Our hospital-based case control study was carried out during the last two years in groups of patients with the diagnosis of COPD and in controls of Tunisian origin. The involved cases and healthy controls were unrelated.

The baseline demographic characteristics of the 416 study subjects are showed in Table 1.

Tow hundred and thirty-four patients were recruited from the service of pneumology in Tahar Sfar University hospital in Mahdia, Tunisia. All patients satisfied the clinical criteria of COPD set down in the Global Strategy for Obstructive Lung Disease (GOLD) guidelines. Inclusion criteria for COPD are the following: chronic airway symptoms and signs such as coughing, breathlessness, wheezing and chronic airway obstruction, defined as a forced expiratory volume in one second (FEV1)/forced vital capacity (FVC) of $<70 \%$ and an FEV1 of $<80 \%$ of the predicted values from spirometric data; and FEV1 reversibility of $<12 \%$ of prebronchodilator FEV1 after inhalation of $200 \mathrm{mg}$ of salbutamol. COPD phenotype exploration was based on chest radiographic and high-resolution computerized tomography (HRCT) density findings. In our study the COPD groups was stratified as carrying or not an emphysematous change. Patients who do not show a Centrolobular emphysematous change were classified as chronic bronchitis. 
Table 1

Demographic characteristics of COPD patients and healthy controls

\begin{tabular}{lccc}
\hline Demographic characteristic & COPD patient $(n=234)$ & Controls $(n=182)$ & $\mathrm{P}$ \\
\hline Age & $61.75 \pm 13.96$ & $56.43 \pm 7.03$ & $0.27^{\mathrm{a}}$ \\
Male:female & $222: 12$ & $173: 9$ & $0.92^{\mathrm{b}}$ \\
BMI & $25.3 \pm 6.7$ & $24.1 \pm 5.2$ & $0.48^{\mathrm{c}}$ \\
Smoking status & & & \\
$\quad$ Never & 15 & 13 & \\
Ex & 102 & 63 & \\
Current & 117 & 106 & \\
Pack years & $51.76 \pm 29.35$ & $47.58 \pm 26.42$ & \\
Geographic origin in Tunisia & & & $0.17^{\mathrm{b}}$ \\
North & 0 & 4 & $0.11^{\mathrm{c}}$ \\
Center & 234 & 178 & $0.21^{\mathrm{b}}$ \\
South & 0 & 0 &
\end{tabular}

BMI: Body mass index, weight $(\mathrm{kg}) /(\text { height }(\mathrm{m}))^{2}$.

Pack years: (number of cigarettes smoked per day x number of years smoked)/20.

${ }^{a}$ Mann-Whitney U-test.

bPearson's $\chi^{2}$ test.

'Student's t test.

Table 2

Clinical characteristics of COPD patients

\begin{tabular}{lc}
\hline Clinical characteristic & Patients $(n=234)$ \\
\hline COPD phenotype & \\
CB & $(127) 54.27 \%$ \\
CLE + PLE & $(107) 45.72 \%$ \\
Spirometry FEV $_{1}$ & $1.19 \pm 0.78$ \\
FEV $_{1} \%$ pred & $46.7 \pm 19.2$ \\
FVC & $1.65 \pm 0.83$ \\
FVC\% pred & $50.9 \pm 16.4$ \\
FEV $1 /$ FVC $\%$ pred & $69.36 \pm 0.66$ \\
RV\% & $4.3 \pm 3.2$ \\
COPD stage & \\
I & (6) $2.56 \%$ \\
II & $(40) 17.09 \%$ \\
III & (113) $48.29 \%$ \\
IV & $(75) 32.05 \%$ \\
\hline
\end{tabular}

CB: Chronic Bronchitis; CLE: Centrolobular Emphysema; PLE: Panlobular Emphysema; $\mathrm{FEV}_{1}$ : Forced Expiratory Volume in $1 \mathrm{sec}$

(L); FVC: Forced Vital Capacity (L); $\mathrm{FEV}_{1}$ $\%$ pred: percentage of the predicted $\mathrm{FEV}_{1}$ value adjusted for age, height and weight. FVC $\%$ pred: percentage of the predicted FVC value adjusted for age, height and weight. RV: Reversibility of baseline $\mathrm{FEV}_{1}$ following bronchodilator inhalation.

The patients with bronchial asthma were excluded on the basis of reversibility of airflow obstruction. The detailed information of patients is listed in Table 2.

The control group contains one hundred and eighty two healthy subjects; all were recruited from a blood donor's cohort from the central area of Tunisia. Individuals with respiratory diseases or any familial history of lung disease were excluded. All control subjects exhibited normal pulmonary function (FEV1/ FVC >
$70 \%$ and FEV1 $>80 \%$ predicted). Hospital ethics committee approval and informed consent were obtained.

For genotyping, DNA was isolated from whole peripheral blood by incubation overnight with proteinase $\mathrm{K}(0.1 \mathrm{mg} / \mathrm{mL})$ in $1 \%$ sodium dodecyl sulfate at $37^{\circ} \mathrm{C}$, extracted with phenol: chloroform and ethanol precipitation as previously described [30].

\subsection{GSTM1 and GSTT1 genotyping}

A multiplex polymerase chain reaction (PCR) was performed to examine the polymorphisms of GSTM1 and GSTT1 as it was described in the literature [31]. PCR for albumin was also performed as an internal control. The primer pairs for each gene were the following: GSTM1:5'-GAACTCCCTGAAAAGCTA AAGC-3', 5'-GTTGGGCTCAAATATACGGTGG-3'; GSTT1: 5'-TTCCTTACTGGTCCTCACATCTC-3', 5' - CAGCTGCATTTGGAAGTGCTC-3' and finally for albumin: 5'-GCCCTCTGCTAACAAGTCCTAC3', 5' GCCCTAAAAAGAAAATCGCCAATC-3'. The PCR buffer contains $20 \mathrm{ng}$ DNA, $1.5 \mathrm{mM} \mathrm{MgCl} 2$, $200 \mathrm{mM}$ of each dNTP, primers of GSTM1 at $3 \mu \mathrm{g} / \mathrm{mL}$ each, primers of GSTT1 at $1 \mu \mathrm{g} / \mathrm{mL}$ each and albumin primers at $1 \mu \mathrm{g} / \mathrm{mL}$ each, $4 \mu \mathrm{L}$ of $5 \mathrm{X}$ colorless GoTaq ${ }^{\circledR}$ Flexi Buffer and 1.5 unit of GoTaq ${ }^{\circledR}$ Flexi DNA polymerase (Promega, USA) in a final volume of $20 \mu \mathrm{L}$. PCRs were carried out with a Mastercycler personal thermal cycler (Eppendorf, Germany).

PCR program consisted of 30 cycles of $94^{\circ} \mathrm{C}$ for 30 seconds, $64^{\circ} \mathrm{C}$ for 30 seconds, $72^{\circ} \mathrm{C}$ for 30 seconds, and a final extension at $72^{\circ} \mathrm{C}$ for five minutes. The initial pre-treatment was carried out at $95^{\circ} \mathrm{C}$ for 5 min- 
utes. The PCR products containing fragments of 215 bp, $480 \mathrm{bp}$ and $350 \mathrm{bp}$ (indicating respectively the presence of GSTM1, GSTT1 and Albumin) were separated by electrophoresis with ethidium bromide stained $2 \%$ agarose gel and visualized by UV detection.

\subsection{PCR-RFLP analysis of GSTP1 gene polymorphisms}

Genotypes were carried out using the method of Ishii et al. with slight modification [21]. For both exon 5 and exon 6, genomic DNA (20 ng) was amplified in $25 \mathrm{~mL}$ reaction mixture containing $1.5 \mathrm{mM}$ $\mathrm{MgCl} 2,1 \mu \mathrm{M}$ of each primer, $200 \mathrm{mM}$ deoxyribonucleoside triphosphate (dNTP) and 0.5 IU of GoTaq ${ }^{\circledR}$ Flexi DNA polymerase (Promega, USA). The following primers: 5'-GTAGTTTGCCCAAGGTCAAG-3' and 5'-AGCCACCTGAGGGGTAAG-3' were used for the amplification of exon 5.

After enzyme digestion with Alw26I (Fermentas, Germany) at $37^{\circ} \mathrm{C}$ during two hours, the homozygous wild genotype yielded two bands, 329 and 104 bp; while homozygous mutant genotype yielded two bands of 222 and 107/104 bp. The two variant alleles in exon 6 were also differentiated by RFLP. The DNA of each sample was subjected to PCR amplification with the following primers: 5'-GG GAGCAAGCAGAGGAGAAT-3' and 5'-CAGGTTG TAGTCAGCGAAGGAG-3'. The restriction enzyme AciI (Fermentas, Germany) was used to digest the PCR product. Wild type genotype shows 247, 118 and 55 bp fragments while mutant genotype shows 365 and 55 bp fragments.

\subsection{Analysis of EPHX1 gene polymorphisms}

Genotypes of exon 3 and 4 EPHX1 polymorphisms were performed by PCR-RFLP analysis similar to that described previously [6]. These primers: EPO1 (5'GATCGATAAGTTCCGTTTCACC-3') and EPO2 (5' ATCCTTAGTCTTGAAGTGAGGAT-3') were used for the amplification of exon 3 . The same reaction mixture as above was used. After enzyme digestion with EcoRV (Fermentas, Germany) for overnight at $37^{\circ} \mathrm{C}$, homozygous wild genotype yielded two bands, 140 and 20 bp; while homozygous mutant genotype yielded only one band of $162 \mathrm{bp}$.

As for exon 4, the primers were as following: EPO3 (5'ACATCCACTTCATCCACGT-3') and EPO4 (5'ATGCCTCTGAGAAGCCAT-3'). The restriction enzyme RsaI (Fermentas, Germany) was used to digest the PCR product. The wild type genotype shows a fragment of $210 \mathrm{bp}$ while mutant genotype yielded two fragments of 164 and $46 \mathrm{bp}$.

\subsection{Lung function measurements}

To assess the lung function impairment, follow-up examinations were conducted with the group of patients during a two-year period after baseline, with annually repeated spirometry test. Complete clinical information and longitudinal lung function measurements were obtained during these two years.

The lung function measurements were carefully standardized and monitored using a high-quality control program to control for multiple factors that were known to increase intraindividual variability of FEV1 and FVC results.

\subsection{Statistical analysis}

Hardy-Weinberg equilibrium tests of EPHX1 and GSTP1 genotype distribution in cases and controls were evaluated by calculating $\chi^{2}$ and $\mathrm{P}$ values. Differences in allele distribution and allele frequencies among the groups were examined for statistical significance by Pearson's $\chi^{2}$ test. Odds ratios (OR) with their corresponding $95 \%$ confidence intervals $(\mathrm{CI})$ were calculated. The $\chi^{2}$ test was used to compare the gender, smoking status and geographic origin between the two groups. Demographic data and spirometric parameters are presented as means \pm standard deviation. Age, smoking index expressed as pack years (number of cigarettes smoked per day $\mathrm{x}$ number of years smoked 120) were compared using the unpaired Student's t test and Mann-Whitney U-test where appropriate. Annual FEV1 decline (mL/year) was calculated as the difference between follow-up and baseline observed FEV1 values, divided by the number of months between the two surveys, multiplied by 12 .

Multivariate logistic regression model was constructed to confirm the association between combined EPHX1, GSTM1, GSTT1 and GSTP1 gene polymorphisms with COPD as well as emphysema by adjustment for covariates, including age, sex, BMI, smoking status and pack years of smoking. Where appropriate, $\mathrm{P}$ values for pairwise differences were corrected for multiple comparisons by using Bonferroni correction with statistical significance set at $P<0.008$. The statistical power was calculated to estimate the level of association.

Multivariate linear regression was also used to confirm the association between combined gene polymorphisms and $\triangle \mathrm{FEV}_{1}$. We used the KolmogorovSmirnov test to test the distribution of $\Delta$ FEV1 variable for normality.All statistical analyses were undertaken using the Statistical Package for the Social Sciences (SPSS), version 10 software. $P<0.05$ was considered as statistically significant. 


\section{Results}

A total of 416 subjects were entered in this casecontrol study: 234 COPD patients (male:female ratio, 222:12; mean age, $61.75 \pm 13.96)$ and 182 healthy controls (male:female ratio, 173:9; mean age, $56.43 \pm$ 7.03). Both patients and controls had a similar bodymass index (BMI; $P=0.48$ ) and were almost all from the central area of Tunisia. There was no significant difference between patients and controls in term of pack years cigarette smoking $(P=0.11)$ (Table 1$)$. Clinical characteristics of patients were summarized in Table 2 . From all cases, $54.27 \%$ had a chronic bronchitis whereas $45.72 \%$ of them had emphysema.

\subsection{Combined effect of EPHX1, GSTP1, M1 and T1 gene polymorphisms and COPD risk}

Genotypes were determined by the combined data obtained from individual PCR-RFLP analysis of exon 3 and 4 EPHX1, exon 5 GSTP1 polymorphisms and PCR multiplex examination of GSTM1 and GSTT1 deletions. As for exon 6 GSTP1 polymorphism, neither heterozygote (Ala/Val) nor homozygous mutants (Val/Val) were found in both patient and control groups, so that it was excluded in the analysis.

The analysed genotype combinations in this study were chosen basing on the principle previous investigations results suggesting that presence of variant alleles in these genes may increase risk of COPD. These genotypes were the following: null-GSTM1/null-GSTT1; 105Val/Val GSTP1/null GSTT1; 113His/His EPHX1/ null GSTT1; 113His/His EPHX1/null-GSTM1; 113His /His EPHX1/105Val/Val GSTP1; null GSTM1/105Val /Val GSTP1.

113His/His EPHX1/null-GSTM1/null-GSTT1/105 Val/Val GSTP1 and 113His/His EPHX1/null-GSTM1/ $105 \mathrm{Val} / \mathrm{Val}$ GSTP1 combined genotypes were not considered because of the lower number of individuals in these subgroups which made ineffective the statistical calculations and interpretations. The distribution of different combinations among healthy controls and patients can be found in Table 3.

In crude combined analysis of EPHX1, GSTP1, GS TM1 and GSTT1 gene polymorphisms, our results demonstrated that combined 113His/His EPHX1/nullGSTM1, null-GSTM1/105Val/Val GSTP1 and 113His/ His EPHX1/105Val/Val GSTP1 genotypes were associated with significantly increased risk of COPD (respectively $\mathrm{OR}=4.07 ; \mathrm{CI}: 1.52-10.87 ; P=0.0032$, $\mathrm{OR}=3.56 ; \mathrm{CI}: 1.46-8.68 ; P=0.0042$ and $\mathrm{OR}=$
3.65; CI:1.1-12.08; $P=0.027)$. When the bonferroni correction for multiple testing was applied, differences of 113His/His EPHX1/null-GSTM1 and null-GSTM1/ 105Val/Val GSTP1 combined genotypes remained significant (respectively $P=0.019 ; P=0.025$ ).

After adjustments for confounding variables, the association between 113His/His EPHX1/null-GSTM1, null-GSTM1/ 105Val/Val GSTP1 combined gene polymorphisms and the increased COPD risk remained significant (respectively $P=0.0094, P=0.0153$ ). As for 113His/His EPHX1/105Val/Val GSTP1 combination, the association with COPD susceptibility was significant in the univariate analysis $(P=0.027)$. After controlling for other COPD confounding factors, the risk decreased slightly and the association became no significant $(P=0.0687)$. Furthermore, our results showed that null-GSTM1/null-GSTT1 $(\mathrm{OR}=1.66$; $\mathrm{CI}$ : 0.95-2.9; $P=0.072), 105 \mathrm{Val} / \mathrm{Val}$ GSTP1/null GSTT1 $(\mathrm{OR}=2.51$; CI: $0.97-6.5 ; P=0.052)$ and $113 \mathrm{His} / \mathrm{His}$ EPHX1/null GSTT1 genotypes $(\mathrm{OR}=2.68$; CI: 0.937.68; $P=0.058$ ) were not related to COPD.

\subsection{EPHX1, GSTP1, M1 and T1 gene polymorphisms combinations and COPD phenotypes}

According to their predominant phenotype, 127 patients exhibited the bronchial type of the disease (chronic bronchitis subgroup), while 107 presented centrolobular and panlobular emphysema. Table 4 summarizes the distribution of combined EPHX1, GSTP1, GSTM1 and GSTT1 genotypes between chronic bronchitis and emphysema COPD patients. The results demonstrated that there was no association between all combined studied genotypes and chronic bronchitis. In the emphysematous group, as for the nullGSTM1/105Val/Val GSTP1, 105Val/Val GSTP1/null GSTT1, 113His/His EPHX1/null-GSTM1 and NullGSTM1/null-GSTT1 combined genotypes in relationship to emphysema, the significance remained after the Bonferroni correction (respectively $P=0.0018 ; P=$ 0.011; $P=0.0018 ; P=0.039$ ).

In the multivariate regression analysis, the combined genotypes null-GSTM1/null-GSTT1 (OR = 2.48; CI: 1.27-4.83), 105Val/Val GSTP1/null GSTT1 (OR = 4.71; CI: 1.61-13.79), 113His/His EPHX1/nullGSTM1 (OR = 6.96; CI: 2.28-21.29) and nullGSTM1/105Val/Val GSTP1 (OR = 5.83; CI: 2.1216.01 ) were found in association with emphysema even after the adjustment for COPD confounding risk factors (respective adjusted $\mathrm{P}$ values: $P=0.01 ; P=0.009$; $P=0.008$ and $P=0.001)$. Whereas the association 
Table 3

Distribution of combined EPHX1, GSTP1, M1 and T1 genotypes in COPD patients and healthy controls

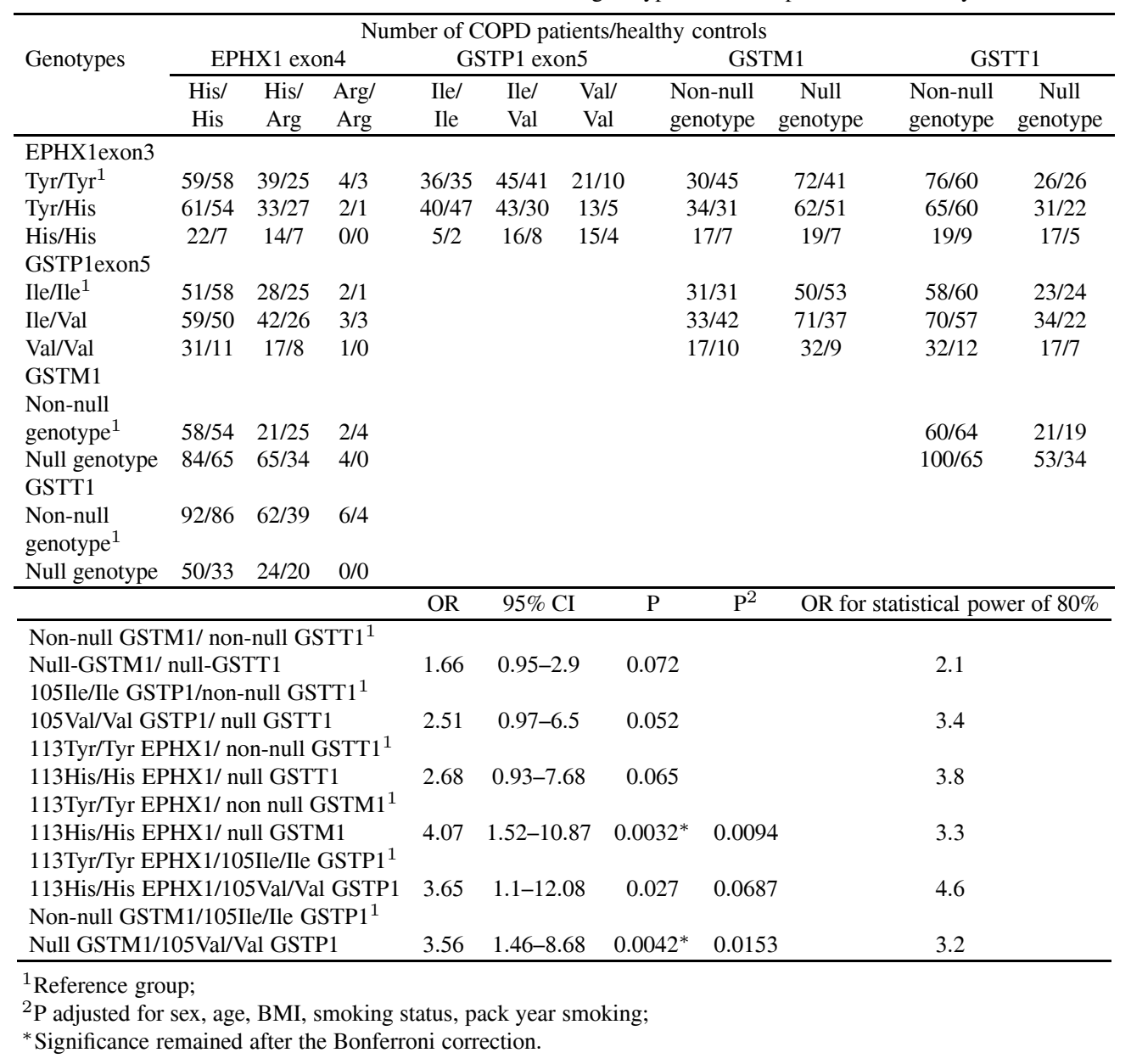

between 113His/His EPHX1/105Val/Val GSTP1 (OR $=4.67$; CI: $1.22-17.91)$ and 113His/His EPHX1/null GSTT1 (OR = 3.33; CI: 1.05-10.52) combined genotypes were attenuated after correction (respective adjusted $P$ value: $P=0.056$ and $P=0.071)$.

\subsection{Association between lung function impairment and EPHX1, GSTP1, M1 and T1 gene polymorphisms}

Annual $\mathrm{FEV}_{1}$ decline means distribution were compared according to different polymorphisms combinations. Combined gene polymorphisms associations with lung function impairment are presented in Table 5. After multivariate linear regression adjusted for COPD confounding factors the obtained results showed that annual FEV1 decline and 113His/His EPHX1/null-GS TM1 $(P=0.028)$ combined genotypes were related. However, the associations between null-GSTM1/ null-
GSTT1, 105Val/Val GSTP1/null GSTT1, 113His/His EPHX1/null GSTT1, 113His/His EPHX1/105Val/Val GSTP1 and null-GSTM1/105Val/Val GSTP1 combinations were not significant (respective $\mathrm{P}$ values: $P=$ 0.097; $P=0.072 ; P=0.081 ; P=0.088$ and $P=$ $0.174)$.

\section{Discussion}

Up to now, more than 25 different candidate genes have been investigated for their potential role in lung function impairment in smokers [32,33]. Few works were interested to study the combinations of polymorphisms in COPD. In this study, we provided a new data about the association between the combined EPHX1, GSTP1, GSTM1 and GSTT1 genetic polymorphisms and COPD risk and lung function impairment in a Tunisian population. Our results suggest that 


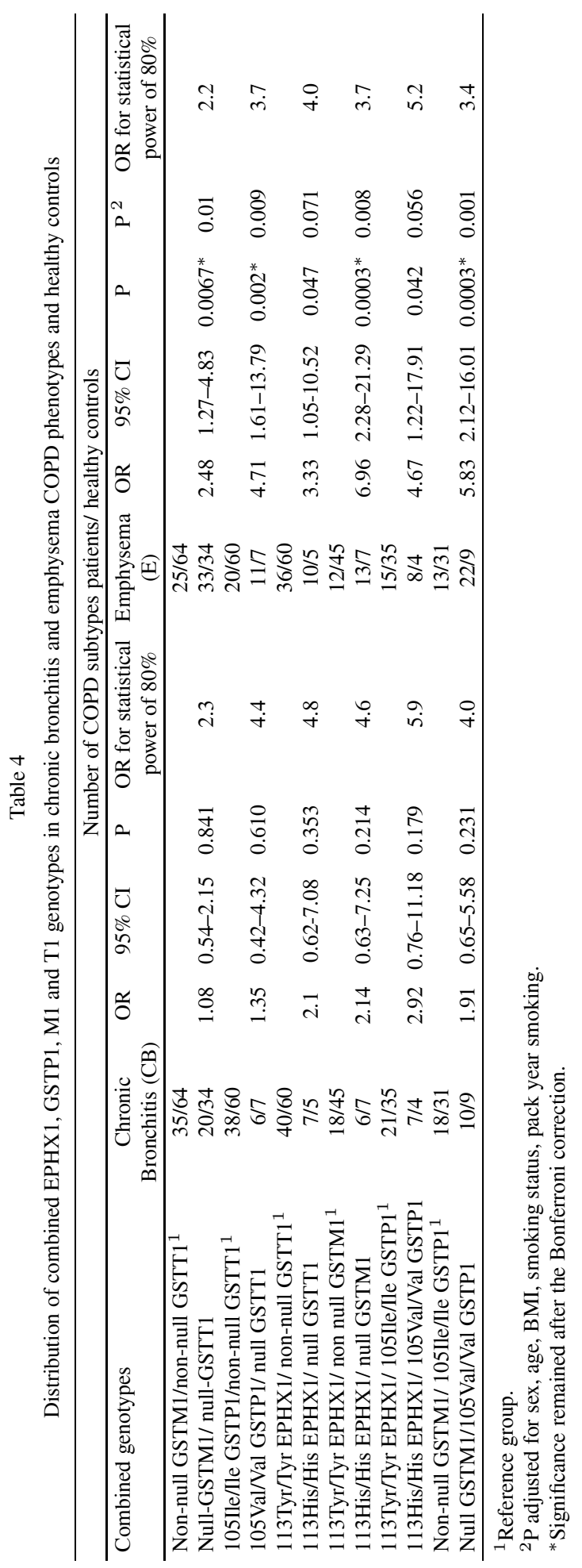


Table 5

Combined EPHX1, GSTP1, M1 and T1 gene polymorphisms association with lung function impairment in COPD patients

\begin{tabular}{lccc}
\hline Combined genotypes & $\Delta$ FEV1 (mL/year) & $\mathrm{P}^{2}$ & $\mathrm{P}^{3}$ \\
\hline Non-null GSTM1/non-null GSTT1 ${ }^{1}$ & $209 \pm 142$ & & \\
Null-GSTM1/ null-GSTT1 & $171 \pm 132$ & 0.097 & \\
105Ile/Ile GSTP1/non-null GSTT1 ${ }^{1}$ & $204 \pm 129$ & & \\
105Val/Val GSTP1/ null GSTT1 & $179 \pm 144$ & 0.072 & \\
113Tyr/Tyr EPHX1/non-null GSTT1 ${ }^{1}$ & $207 \pm 153$ & & \\
113His/His EPHX1/null GSTT1 & $176 \pm 137$ & 0.081 & \\
113Tyr/Tyr EPHX1/non null GSTM1 ${ }^{1}$ & $206 \pm 161$ & & \multirow{2}{*}{0.028} \\
113His/His EPHX1/null GSTM1 & $144 \pm 101$ & 0.035 & \\
113Tyr/Tyr EPHX1/105Ile/Ile GSTP1 ${ }^{1}$ & $205 \pm 216$ & & \\
113His/His EPHX1/105Val/Val GSTP1 $^{1}$ & $174 \pm 169$ & 0.088 & \\
Non-null GSTM1/105Ile/Ile GSTP1 ${ }^{1}$ & $199 \pm 136$ & & \\
Null GSTM1/105Val/Val GSTP1 & $178 \pm 156$ & 0.174 & \\
\hline${ }^{1}$ Reference group. & & & \\
${ }^{2}$ Student's t test. & & & \\
${ }^{3}$ P adjusted for sex, age, BMI, smoking status, pack year smoking. & &
\end{tabular}

the combined 113His/His EPHX1/null-GSTM1, nullGSTM1/105Val/Val GSTP1 genotypes are associated with significantly increased risk of COPD. 113His/His EPHX1/105Val/Val GSTP1 association with the disease was significant before adjustment for COPD risk factors.

Our findings are in line with some studies reporting that the odds ratio was raised when the two genotypes of at least one slow EPHX1 exon-3 allele and GSTM1 null were combined $[12,13]$. The present genes are situated on different chromosomes and linkage disequilibrium is not likely. However, each locus could be in linkage disequilibrium with an unknown casual gene(s). The potential for linkage disequilibrium is a fundamental limitation of the candidate gene association approach and depends on the linkage disequilibrium surrounding each locus in the study population. Therefore, functional studies, including analysis of EPHX1 and GST messenger ribonucleic acid and protein expression, should be continued to confirm the causal relationship before conclusions can definitely be drawn. Nevertheless, the replication of the results in several works could be a sign that there was still a significant trend towards association between these gene polymorphisms and the development of COPD $[12,13]$.

Furthermore, gene frequency is different between different racial groups, which may explain the differences in association between gene polymorphism and COPD in the various studies. In fact, the distribution of EPHX1 and GST genotypes are complex among races $[10,13,34,35]$ and the difference in genotype frequencies might be relevant to different metabolising enzyme activities and types of dominant functional enzymes against oxidative stress in different races.
The evidence of association between the candidate genes and COPD and its associated phenotypes is seldom definitive and is often conflicting [36]. Smith and Harrison (1997) reported first that EPHX1 gene polymorphisms were associated with susceptibility to pulmonary emphysema. There has been also some evidence that GSTM1 null genotype might be related to severe bronchitis in heavy smokers [20] and to reduction in lung function and emphysema in smokers with bronchogenic carcinoma [19,37]. Moreover, DeMeo and co-authors reported an association of GSTP1 and EPHX1 gene polymorphisms with emphysema distribution [38].

In the present study, we have analyzed combined genotypic data within patients divided into chronic bronchitis and emphysema subgroups. Our results suggested a significant association between 113His/His EPHX1/null-GSTM1 and null-GSTM1/105Val/Val GS TP1 genotypes with emphysema. The association between 113His/His EPHX1/105Val/Val GSTP1 and this subgroup was slightly attenuated after adjustment for covariates. The majority of glutathione S-transferase activity in the lung is likely provided by GSTP1 [39]. The EPHX1 enzyme activity has been detected in all tissues, and the highest concentrations have been found in lung, liver, kidney, gonads, and epithelial cells [40]. Several recent studies were focused on the relationship between gene polymorphisms and lung function in COPD patients, smokers and in general population with controversial results [41-47].

In our present investigations, we explored the relationships between EPHX1, GSTM1, GSTT1, GSTP1 gene polymorphisms combinations and lung function decline in our sample. On the bases of the results found 
we deduce that 113His/His EPHX1/null-GSTM1 combined genotype was in association with the lung function impairment. Our findings are in agreements with those of Vibhuti and colleagues. The authors found an association between EPHX1 and GSTP1 genotypes and lung function either when studied separately or in combination [46]. Andrew and co-authors demonstrated that EPHX1 His113/His139 haplotype was associated with increased rate of decline of lung function. This association was stronger when the subjects also had a family history of COPD [6]. JianQing and colleagues reported that concurrent deletion of GSTT1 and GSTM1 genes together with the presence of the GSTP1 105Ile/Ile genotype carried an increased risk for accelerated decline of lung function in smokers. In addition, the combination of homozygous GSTP1 105Ile/Ile and a family history of COPD were associated with an increased risk of decline of lung function [48]. Imboden and co-workers found that GSTT1 homozygous gene deletion in combination with GSTM1 homozygous gene deletion was associated with excess decline in FEV1 in men general population [49]. In our sample, COPD family history was absent. The presence of more than one gene polymorphism seems to be with main effect of the lung decline in the studied population. A long longitudinal study and a larger population are of a great importance to draw firm conclusions. By contrast, Siedlinski et al. recently reported that there are no effects of EPHX1 polymorphisms on the level or change of FEV1 in the general population [47]. Indeed the contribution of EPHX1 polymorphisms on COPD susceptibility is controversial and the discrepancy between studies may be due to the different ethnic groups investigated.

There are several limitations of the present study. First, a relatively small population was recruited and the numbers of males and females were not balanced within the two studied groups. The major population of COPD patients and chronic smokers is male in Tunisia. Therefore, it is difficult to find a balanced set of female subjects for genotype studies. Secondly, there were a difference of the mean age between cases and controls. In fact Younger controls were more likely to provide a blood sample than older controls and were over sampled. However, the statistical calculation showed that there is no significant difference between the two groups in mean age, so it is unlikely that this age difference confounded the results. Additionally, age was included in all the multivariate models. Thirdly, after patients classification according to COPD phenotypes, and genotype combinations the size of the subgroups became small and this may affect the statistical power and so that our findings might be considered with caution.

In conclusion, we have demonstrated that combined genotypes 113His/His EPHX1/ null-GSTM1 and null-GSTM1/ 105Val/Val GSTP1 were in association with the development of COPD in the Tunisian population. A significant association was also found between these genotypes and emphysema. Moreover 113His/His EPHX1/ null-GSTM1 combined genotype was in relationship with lung function impairment. Further works using a larger population and studying more candidate genes taking in account the gene- environment interaction remains necessary in order to elucidate the genetic pathogenesis of COPD.

\section{Acknowledgements}

The authors thank Pr. A. MILED and Dr. A. KASSAB from Biochemistry Laboratory, CHU Farhat Hached, Sousse, Tunisia for their suggestions and helpful comments.

\section{References}

[1] B.R. Celli, W. MacNee and ATS/ERS, Task Force Standards for the diagnosis and treatment of patients with COPD: a summary of the ATS/ERS position paper, Eur Respir $J \mathbf{2 3}$ (2004), 932-946.

[2] A. Lokke, P. Lange, H. Scharling, P. Fabricius and J. Vestbo, Developing COPD: a 25 year follow up study of the general population, Thorax 61 (2006), 935-939.

[3] R. Bascom, Differential susceptibility to tobacco smoke: possible mechanisms, Pharmacogenetics 1 (1991), 102-106.

[4] R. Ugenskiene, M. Sanak, R. Sakalauskas and A. Szczeklik, Genetic polymorphisms in chronic obstructive pulmonary disease, Medicina (Kaunas) 41 (2005), 17-22.

[5] N. Hizawa, Genetic backgrounds of asthma and COPD, Allergol Int 58 (2009), 315-322.

[6] C.A. Smith and D.J. Harrison, Association between polymorphism in gene for microsomal epoxide hydrolase and susceptibility to emphysema, Lancet 350 (1997), 630-633.

[7] A.J. Sandford, T. Chagani, T.D. Weir, J.E. Connett, N.R. Anthonisen and P.D. Pare, Susceptibility genes for rapid decline of lung function in the lung health study, Am J Respir Crit Care Med 163 (2001), 469-473.

[8] J.Y. Park, L. Chen, N. Wadhwa and M.S. Tockman, Polymorphisms for microsomal epoxide hydrolase and genetic susceptibility to COPD, Int J Mol Med 15 (2005), 443-448.

[9] C.P. Hersh, D.L. De Meo, C. Lange, A.A. Litonjua, J.J. Reilly, D. Kwiatkowski, N. Laird, J.S. Sylvia, D. Sparrow, F.E. Speizer, S.T. Weiss and E.K. Silverman, Attempted replication of reported chronic obstructive pulmonary disease candidate gene associations, Am J Respir Cell Mol Biol 33 (2005), $71-78$. 
[10] J.J. Yim, G.Y. Park, C.T. Lee, Y.W. Kim, S.K. Han, Y.S. Shim and C.G. Yoo, Genetic susceptibility to chronic obstructive pulmonary disease in Koreans: combined analysis of polymorphic genotypes for microsomal epoxide hydrolase and glutathione S-transferase M1 and T1, Thorax 55 (2000), 121125.

[11] K. Takeyabu, E. Yamaguchi, I. Suzuk, M. Nishimura, N. Hizawa and Y. Kamakami, Gene polymorphism for microsomal epoxide hydrolase and susceptibility to emphysema in a Japanese population, Eur Respir J 15 (2000), 891-894.

[12] S.L. Cheng, C.J. Yu, C.J. Chen and P.C. Yang, Genetic polymorphism of epoxide hydrolase and glutathione S-transferase in COPD, Eur Respir J 23 (2004), 818-824.

[13] J. Zidzik, E. Slabá, P. Joppa, Z. Kluchová, Z. Dorková, P. Skyba, V. Habalová, J. Salagovic and R. Tkácová, Glutathione S-transferase and Microsomal Epoxide Hydrolase Gene Polymorphisms and Risk of Chronic Obstructive Pulmonary Disease in Slovak Population, Croat Med J 49 (2008), 182-191.

[14] J. Lee, B.G. Nordestgaard and M. Dahl, EPHX1 polymorphisms, COPD and asthma in 47,000 individuals and in meta-analysis, Eur Respir J (2010), doi: 10.1183/09031936. 00012110 .

[15] B. Ketterer, D.J. Meyer and A.G. Clark, Soluble glutathione transferase isoenzymes. In: Sies H, Ketterer B, eds., Glutathione conjugation, London: Academic Press (1988), 357386.

[16] J.O. Curtis, C. Hassett and V. Hosagrahara, Epoxide hydrolase - polymorphism and role in toxicologym Toxicol Lett 112 (2000) 365-370

[17] C. Hassett, L. Aicher, J.S. Sidhu and C.J. Omiecinski, Human microsomal epoxide hydrolase: genetic polymorphism and functional expression in vitro of amino acid variants, Hum Mol Genet 3 (1994), 421-428.

[18] A.S. Johansson, G. Stenberg, M. Widersten and B. Mannervik, Structure activity relationships and thermal stability of human glutathione transferase $\mathrm{P} 1-1$ governed by the $\mathrm{H}$-site residue 105, J Mol Biol 278 (1998), 687-698.

[19] D.J. Harrison, A.M. Cantlay, F. Rae, D. Lamb and C.A. Smith, Frequency of glutathione S transferase M1 deletion in smokers with emphysema and lung cancer, Hum Exp Toxicol 16 (1997), 356-360.

[20] H. Baranova, J. Perriot, E. Albuisson, T. Ivaschenko, V.S. Baranov, B. Hemery, P. Mouraire, N. Riol and P. Malet, Peculiarities of the GSTM1 0/0 genotype in French heavy smokers with various types of chronic bronchitis, Hum Genet 99 (1997), 822-826.

[21] T. Ishii, T. Matsuse, S. Teramoto, H. Matsui, M. Miyao, T. Hosoi, H. Takahashi, Y. Fukuchi and Y. Ouchi, Glutathione Stransferase P1 (GSTP1) polymorphism in patients with chronic obstructive pulmonary disease, Thorax 54 (1999), 693-696.

[22] D. Xiao, C. Wang, M.J. Du, B.S. Pang, H.Y. Zhang, B. Xiao, J.Z. Liu, X.Z. Weng, L. Su and D.C. Christiani, Relationship between polymorphisms of genes encoding microsomal epoxide hydrolase and glutathione S-transferase P1 and chronic obstructive pulmonary disease, Chin Med J 117 (2004), 661667.

[23] F. Rodríguez, C. de la Roza, R. Jardi, M. Schaper, R. Vidal and M. Miravitlles, Glutathione S-Transferase P1 and Lung Function in Patients With $\alpha 1$-Antitrypsin Deficiency and COPD, Chest 127 (2005), 1537-1543.

[24] J. Smolonska, C. Wijmenga, D.S. Postma and H.M. Boezen, Meta-analyses on suspected chronic obstructive pulmonary disease genes: a summary of 20 years' research, Am J Respir Crit Care Med 180 (2009), 618-631.
[25] F. Yan, C. Chen, J. Jing, W. Li, H. Shen and X. Wang, Association between polymorphism of glutathione S-transferase P1 and chronic obstructive pulmonary disease: a meta-analysis, Respir Med 104 (2010), 473-480.

[26] P.J. Castaldi, M.H. Cho, M. Cohn, F. Langerman, S. Moran, N. Tarragona, H. Moukhachen, R. Venugopal, D. Hasimja, E. Kao, B. Wallace, C.P. Hersh, S. Bagade, L. Bertram, E.K. Silverman and T.A. Trikalinos, The COPD genetic association compendium: a comprehensive online database of COPD genetic associations, Hum Mol Genet 19 (2010), 526-534.

[27] R. Lakhdar, S. Denden, J. Knani, N. Leban, H. Daimi, M. Hassine, G. Lefranc, J. Ben Chibani and A. Haj Khelil, Association between GST M1 and GSTT1 polymorphisms with COPD in Tunisian population, J Biochem Genet 48 (2010), 647-657.

[28] R. Lakhdar, S. Denden, J. Knani, N. Leban, H. Daimi, M. Hassine, G. Lefranc, J. Ben Chibani and A. Haj Khelil, Relationship between glutathione S-transferase P1 polymorphisms and chronic obstructive pulmonary disease in Tunisian population, Genet Mol Res 9 (2010), 897-907.

[29] R. Lakhdar, S. Denden, J. Knani, N. Leban, H. Daimi, M. Hassine, G. Lefranc, J. Ben Chibani and A. Haj Khelil, Microsomal epoxide hydrolase gene (EPHX1) polymorphisms and susceptibility to chronic obstructive pulmonary disease in Tunisian population, Genet Test Mol Biomarkers DOI: 10.1089/gtmb.2009.0168.

[30] L.G. Dsavis, M.D. Dibner and J.F. Batte, Basic methods in molecular biology. Elsevier, New York (1986), 44-87.

[31] M. Arand, R. Muhlbauer, J. Hengstler, E. Jäger, J. Fuchs, L. Winkler and F. Oesch, A multiplex polymerase chain reaction protocol for the simultaneous analysis of the glutathione S-transferase GSTM1 and GSTT1 polymorphisms, Anal Biochem 236 (1996), 184-186.

[32] N.A. Molfino, Genetics of COPD, Chest 125 (2004), 19291940.

[33] L. Joos, J.Q. He, M.B. Shepherdson, J.E. Connett, N.R. Anthonisen, P.D. Pare and A.J. Sandford, The role of matrix metalloproteinase polymorphisms in the rate of decline in lung function, Hum Mol Genet 11 (2002), 5679-5676.

[34] H.H. Nelson, J.K. Wiencke, D.C. Christiani, T.J. Cheng, Z.F. Zuo, B.S. Schwartz, B.K. Lee, M.R. Spitz, M. Wang, X. Xu et al., Ethnic differences in the prevalence of the homozygous deleted genotype of glutathione S-transferase theta, Carcinogenesis 16 (1995), 1243-1245.

[35] C.L. Chen, Q. Liu and M.V. Relling, Simultaneous characterization of glutathione S-transferase M1 and T1 polymorphisms by polymerase chain reaction in American whites and blacks, Pharmacogenetics 6 (1996), 187-191.

[36] E.K. Silverman, Progress in chronic obstructive pulmonary disease genetics, Proc Am Thorac Soc 3 (2006), 405-408.

[37] R. Tkacova, J. Salagovic, M. Ceripkova, I. Tkac, J. Stubna and I. Kalina, Glutathione S-transferase M1 gene polymorphism is related to COPD in patients with non-small-cell lung cancer, Wien Klin Wochenschr 116 (2004), 131-134.

[38] D.L. DeMeo, C.P. Hersh, E.A. Hoffman, A.A. Litonjua, R. Lazarus, D. Sparrow, J.O. Benditt, G. Criner, B. Make, F.J. Martinez, P.D. Scanlon, F.C. Sciurba, J.P. Utz, J.J. Reilly and E.K. Silverman, Genetic Determinants of Emphysema Distribution in the National Emphysema Treatment Trial, Am J Respir Crit Care Med 176 (2007), 42-48.

[39] A.A. Fryer, R. Hume and R.C. Strange, The development of glutathione S-transferase and glutathione peroxidase activities in human lung, Biochim Biophys Acta 883 (1986), 448-453. 
[40] J. Seidegard and G. Ekstrom, The role of human glutathione transferases and epoxide hydrolases in the metabolism of xenobiotics, Environ Health Perspect 105 (1997), 791-799.

[41] G.M. Hunninghake, M.H. Cho, Y. Tesfaigzi, M.E. SotoQuiros, L. Avila, J. Lasky-Su, C. Stidley, E. Melén, C. Söderhäll, J. Hallberg, I. Kull, J. Kere, M. Svartengren, G. Pershagen, M. Wickman, C. Lange, D.L. Demeo, C.P. Hersh, B.J. Klanderman, B.A. Raby, D. Sparrow, S.D. Shapiro, E.K. Silverman, A.A. Litonjua, S.T. Weiss and J.C. Celedón, MMP12, lung function, and COPD in high-risk populations, $N$ Engl J Med 361 (2009), 2599-2608.

[42] E. Repapi, I. Sayers, L.V. Wain, P.R. Burton, T. Johnson, M. Obeidat, J.H. Zhao, A. Ramasamy, G. Zhai, V. Vitart, J.E. Huffman, W. Igl, E. Albrecht, P. Deloukas, J. Henderson, R. Granell, W.L. McArdle, A.R. Rudnicka, Wellcome Trust Case Control Consortium, I. Barroso, R.J. Loos, N.J. Wareham, L. Mustelin, T. Rantanen, I. Surakka, M. Imboden, H.E. Wichmann, I. Grkovic, S. Jankovic, L. Zgaga, A.L. Hartikainen, L. Peltonen, U. Gyllensten, A. Johansson, G. Zaboli, H. Campbell, S.H. Wild, J.F. Wilson, S. Gläser, G. Homuth, H. Völzke, M. Mangino, N. Soranzo, T.D. Spector, O. Polasek, I. Rudan, A.F. Wright, M. Heliövaara, S. Ripatti, A. Pouta, A.T. Naluai, A.C. Olin, K. Torén, M.N. Cooper, A.L. James, L.J. Palmer, A.D. Hingorani, S.G. Wannamethee, P.H. Whincup, G.D. Smith, S. Ebrahim, T.M. McKeever, I.D. Pavord, A.K. MacLeod, A.D. Morris, D.J. Porteous, C. Cooper, E. Dennison, S. Shaheen, S. Karrasch, E. Schnabel, H. Schulz, H. Grallert, N. Bouatia-Naji, J. Delplanque, P. Froguel, J.D. Blakey; NSHD Respiratory Study Team, J.R. Britton, R.W. Morris, J.W. Holloway, D.A. Lawlor, J. Hui, F. Nyberg, M.R. Jarvelin, C. Jackson, M. Kähönen, J. Kaprio, N.M. ProbstHensch, B. Koch, C. Hayward, D.M. Evans, P. Elliott, D.P. Strachan, I.P. Hall and M.D. Tobin, Genome-wide association study identifies five loci associated with lung function, Nat Genet 42 (2010), 36-44.

[43] M. Siedlinski, D.S. Postma, J.M. Boer, G. van der Steege, J.P. Schouten, H.A. Smit and H.M. Boezen, Level and course of FEV1 in relation to polymorphisms in NFE2L2 and KEAP1 in the general population. Respir Res 10 (2009), 73.

[44] C.E Stewart, I.P. Hall, S.G. Parker, M.F. Moffat, A.J. Wardlaw, M.J. Connolly, C. Ruse and I. Sayers, PLAUR polymorphisms and lung function in UK smokers, BMC Med Genet 10 (2009), 112.

[45] A. Kaparianos, E. Argyropoulou, G. Efremidis, C. Flordellis and K. Spiropoulos, Decline in FEV1 related to genetic polymorphisms (+138insA/delA and Lys198Asn) of the endothelin-1 gene in COPD. A pilot study, Eur Rev Med Pharmacol Sci 14 (2010), 705-719.

[46] A. Vibhuti, E. Arif, D. Deepak, B. Singh and M.A. Qadar Pasha, Genetic polymorphisms of GSTP1 and mEPHX correlate with oxidative stress markers and lung function in COPD, Biochem Biophys Res Commun 359 (2007), 136-142.

[47] M. Siedlinski, D.S. Postma, H.A. Smit and H.M. Boezen, No effects of EPHX1 polymorphisms on the level or change of FEV1 in the general population, Eur Respir J 33 (2009), 446-449.

[48] J.Q. He, J. Ruan, J.E. Connett, N.R. Anthonisen, P.D. Paré and A.J. Sandford, Antioxidant Gene Polymorphisms and Susceptibility to a Rapid Decline in Lung Function in Smokers, Am J Respir Crit Care Med 166 (2002), 323-328.

[49] M. Imboden, S.H. Downs, O. Senn, G. Matyas, O. Brändli, E.W. Russi, C. Schindler, U. Ackermann-Liebrich, W. Berger, N.M. Probst-Hensch and SAPALDIA Team, Glutathione S-transferase genotypes modify lung function decline in the general population: SAPALDIA cohort study, Respir Res $\mathbf{8}$ (2007), 2. 


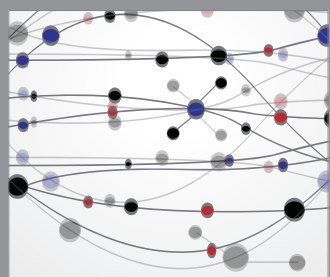

The Scientific World Journal
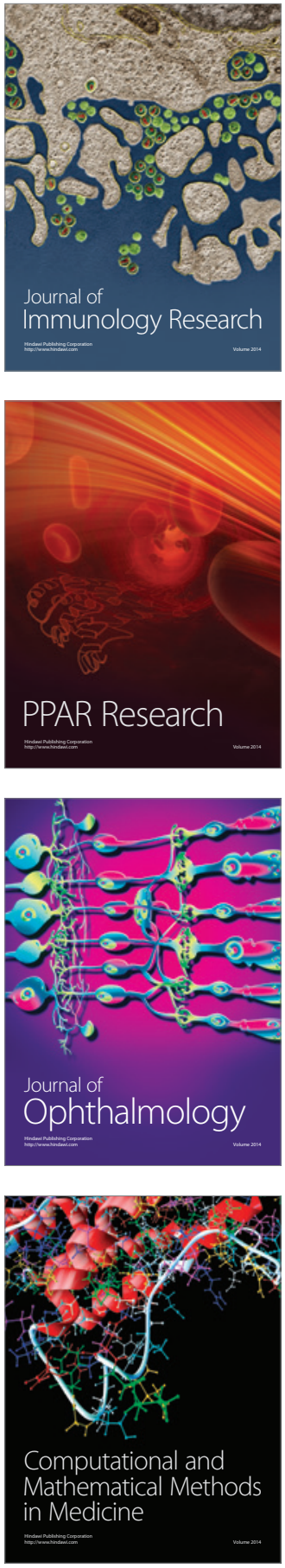

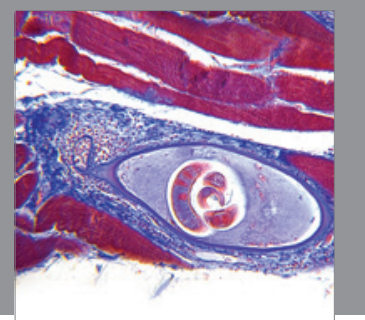

Gastroenterology

Research and Practice
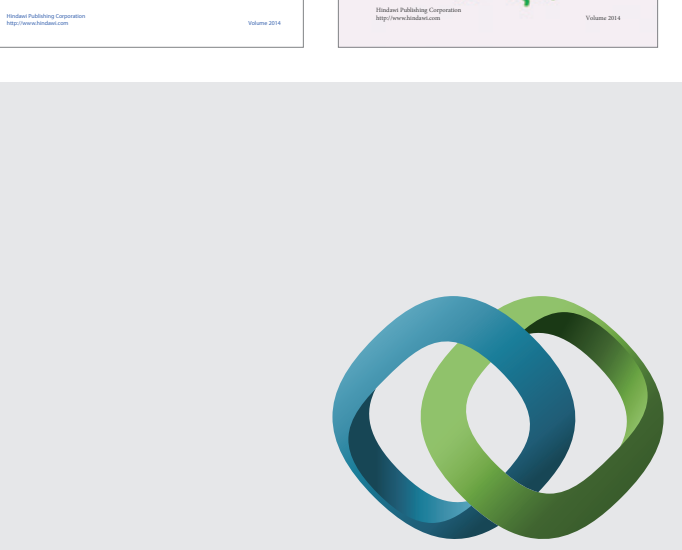

\section{Hindawi}

Submit your manuscripts at

http://www.hindawi.com
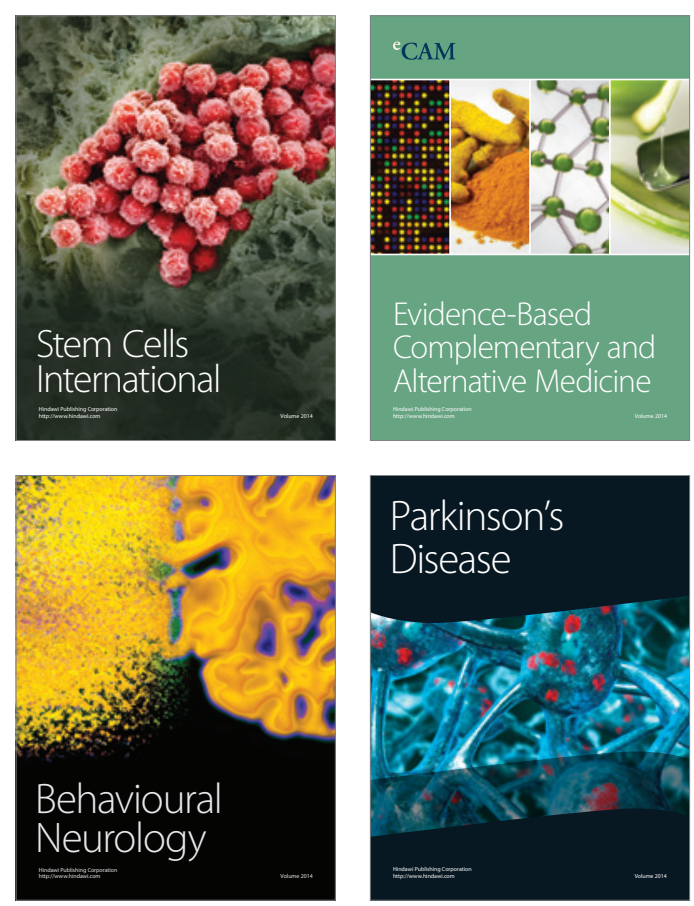

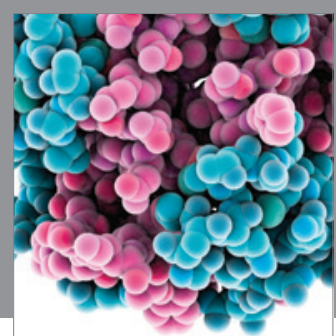

Journal of
Diabetes Research

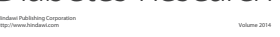

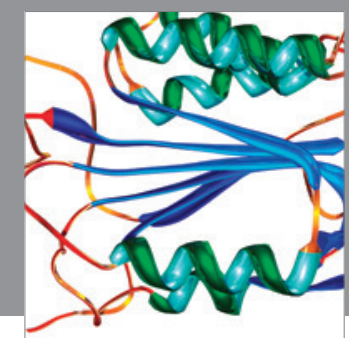

Disease Markers
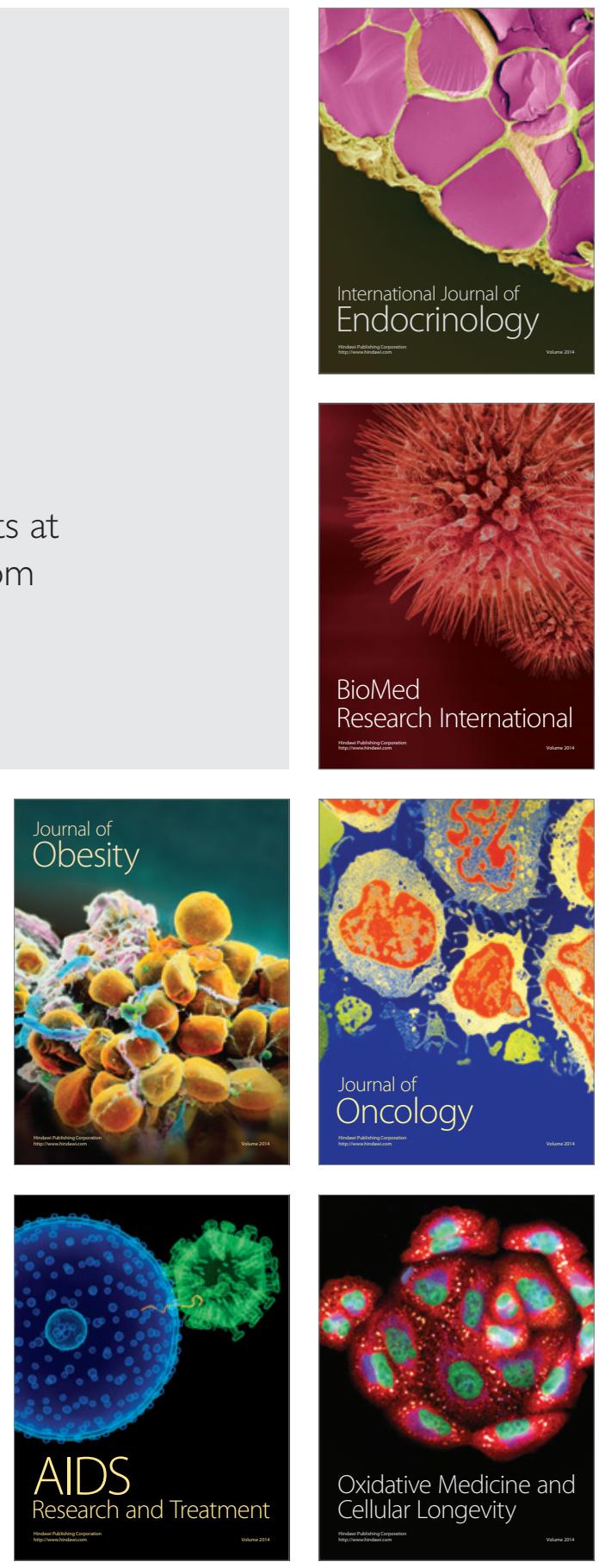